\title{
Modelling ternary effects on antiphase boundary energy of $\mathrm{Ni}_{3} \mathrm{Al}$
}

\author{
K.V. Vamsi ${ }^{\mathrm{a}}$ and S. Karthikeyan \\ Department of Materials Engineering Indian Institute of Science, Bangalore-560012, India
}

\begin{abstract}
The shearing of ordered $\gamma^{\prime}$ precipitates by matrix dislocations results in the formation of antiphase boundaries (APB) in Ni-base superalloys. The APB energy is an important source of order-strengthening in disk and blade alloys where $\mathrm{Ti}$ and $\mathrm{Ta}$ substitute for $\mathrm{Al}$ in $\gamma^{\prime}$. While the importance of APB energy is wellacknowledged, the effect of alloying on APB energy is not fully understood. In the present study, the effect of Ti and Ta additions on the $\{111\}$ and $\{010\}$ APB energies was probed via electronic structure calculations. Results suggest that at low levels of Ti/Ta, APB energies on either plane increases with alloying. However, at higher Ti/Ta levels, the APB energies decrease with alloying. These trends understood by accounting for nearest neighbour violations about the APB and additionally, invoking the effect of precipitate composition on the energy penalty of the violations. We propose an Environment Dependent Nearest Neighbour Bond (EDNNB) model that predicts APB energies that are in close agreement to calculated values.
\end{abstract}

\section{Introduction}

Ni-based disk and blade alloys exhibit excellent high temperature mechanical properties due to the presence of coherent $\gamma^{\prime}$ precipitates in $\gamma$, the Ni-rich f.c.c matrix [1,2]. The higher strength of the precipitates compared to the matrix is a consequence of these precipitates being ordered in an $\mathrm{L1}_{2}$ structure. The primary glide dislocation in $\gamma$ matrix has a Burgers vector of $1 / 2\langle 10-1\rangle$, and these dislocations are glissile on the $\{111\}$ at all temperatures [2]. The $1 / 2\langle 10-1\rangle$ vector is, however, not a lattice translation in $\gamma^{\prime}$ and thus if a dislocation with this Burgers vector cuts through the precipitate along the $\{111\}$ plane, then it would result in the formation of an Anti-Phase Boundary $\left(\mathrm{APB}_{(111)}\right)$. The penalty associated with the creation of this planar fault is the primary source of the strength of the precipitate; higher the APB energy, higher is the strength of the precipitate [2-4]. Due to this, high temperature deformation of Ni-base superalloys involving shear of precipitates, is possible only at high stresses in both in disk and blade alloys [5-8].

The second source of strength of these precipitates is manifested in the form of yield anomaly in Ni-base superalloys, wherein the precipitate strength increases with temperature. This is due to cross-slip of screw segments of dislocations onto a $\{010\}$ plane at high temperatures resulting in the formation of an APB on the cube plane. Since the APB on the $\{010\}$ plane, i.e. $\mathrm{APB}_{(010)}$ has a lower energy than $\mathrm{APB}_{(111)}$, a sessile low energy configuration called the Kear-Wilsdorf locks forms which leads to the yield anomaly [9]. The ratio of the $\mathrm{APB}_{(010)}$ and $\mathrm{APB}_{(111)}$, plays a crucial role on the formation of these locks. Besides $\mathrm{APB}_{(010)}$ and $\mathrm{APB}_{(111)}$, other faults such as the Complex Stacking Fault $\left(\mathrm{CSF}_{(111)}\right)$, Superlattice

\footnotetext{
${ }^{a}$ Corresponding author: karthik@materials.iisc.ernet.in
}

Extrinsic Stacking Fault $\left(\operatorname{SESF}_{(111)}\right)$ and Superlattice Intrinsic Stacking Fault $\left(\mathrm{SISF}_{(111)}\right)$, and their energies also influence, among other things, the precipitate strength, the configuration of extended super-dislocations and the ease of twinning [5].

The energy penalty involved in creation for these faults is well recognized to be a key parameter in determining the high temperature mechanical response of the the $\gamma^{\prime}$ precipitate and so the superalloy [10]. As a result, there exists significant literature on experimental or computational evaluation of fault energies in $\mathrm{Ni}_{3} \mathrm{Al}$ [11-22]. However, such data is not readily available for compositions of $\gamma^{\prime}$ precipitates that deviate from $\mathrm{Ni}_{3} \mathrm{Al}$. This is relevant because Ni-base superalloys are heavily alloyed (up to 15 elements) and several of these elements partition to $\gamma^{\prime}$ and thus modify its composition. As a consequence, the composition of the $\gamma^{\prime}$ is better expressed as $(\mathrm{Ni}, \mathrm{M})_{3}(\mathrm{Al}, \mathrm{X})$, where $\mathrm{M}$ and $\mathrm{X}$ are alloying additions that partitions to $\gamma$ where they substitutes for $\mathrm{Ni}$ and $\mathrm{Al}$. The most important of Al substituting alloying elements (i.e., $\mathrm{X}$ ) are Ta in single crystal blade alloys, and $\mathrm{Ti}$ and $\mathrm{Nb}$ in polycrystalline disk alloys [2].

Only a few studies exist on evaluation of fault energies in the complex compositions [16-18,21,22]. A systematic study on the effect of $\mathrm{Ta}, \mathrm{Ti}$ and $\mathrm{Nb}$ on energy of $\mathrm{APB}_{(111)}$ in $\mathrm{Ni}_{3} \mathrm{Al}_{1-x} \mathrm{X}_{x}$ was undertaken by Chandran et al. [20]. A recent comprehensive study on the effect of $\mathrm{Ta}, \mathrm{Ti}$ and $\mathrm{Ni}$ antisite substitutions in the Al sublattice, on lattice parameters, cohesive energies and energies of $\mathrm{APB}_{(111)}, \mathrm{APB}_{(010)}, \mathrm{CSF}_{(111)}$ and $\mathrm{SISF}_{(111)}$ was conducted by Vamsi et al. [23] in $\mathrm{Ni}_{3} \mathrm{Al}_{(1-x)} \mathrm{X}_{x}$. Addition of $\mathrm{Ta}$ and $\mathrm{Ti}$ resulted in an increase of lattice parameters and decrease in cohesive energies in $\mathrm{Ni}_{3} \mathrm{Al}_{(1-x)} \mathrm{X}_{x}$. whereas $\mathrm{Ni}$ addition lead to decrease and increase in lattice parameters and cohesive energies. Addition of $\mathrm{Ta}$ and $\mathrm{Ti}$, increases the 
stacking fault energies initially and then decreases it at high levels of $\mathrm{X}$. In contrast, addition of $\mathrm{Ni}$, decreases the stacking fault energies monotonically. The observed trends in $\mathrm{APB}_{(111)}$ energy, for the case of $\mathrm{Ta}$ and $\mathrm{Ti}$ were similar to that of the trends observed by Chandran et al. [20]. Additionally, the authors developed a quasichemical model predicting a second order polynomial dependence of fault energies on composition in the dilute regime (for $\mathrm{x}<0.25$ ) [23].

The aim of this study was to build on the previous work [23] and develop a more comprehensive model for predicting the effect of ternary additions on $\mathrm{APB}_{(111)}$ and $\mathrm{APB}_{(010)}$, energies in $\mathrm{Ni}_{3} \mathrm{Al}_{(1-x)} \mathrm{X}_{x}$, where $\mathrm{X}=\mathrm{Ta}$, $\mathrm{Ti}$ over a wider range of composition (for $\mathrm{x}=0-1$ ). The model improves on the previous one [23] by not only accounting for nearest neighbor violations on the fault but by also including the effect of far-field composition. This was done by conducting critical first principles electronic structure calculations to isolate the two effects, and these are explained in the next section. The model development and a comparison between model predictions and calculated values are presented next. The broad implications of the model are then discussed.

\section{Simulation methodology}

\subsection{Computational details}

First principles calculations were done using density functional theory to calculate the energies of the faulted and unfaulted structures. Vienna Ab-initio Simulation Package (VASP 4.6) [24-27] operated within the MedeA ${ }^{\circledR}$ platform [28] was used. Projected augmented wave scheme was used to represent the wave functions. K-point spacing of $0.19-0.20 \AA^{-1}$ and plane wave kinetic energy cutoff of $400 \mathrm{eV}$ were found to be optimal. Methfessel-Paxton first order spreading was used for Brillouin zone integration [29] with $0.127 \mathrm{eV}$ as the smearing width. Pseudopotentials used were based on Generalized Gradient Approximation (GGA-PBE) and of Perdew-Burke-Ernzerhof type [30,31]. Atomic relaxation was performed using a conjugate gradient algorithm and calculations were terminated when Hellmann-Feynman forces were less than $0.02 \mathrm{eV} / \AA$. These values have been previously shown to be robust in predicting bulk properties of $\mathrm{Ni}, \mathrm{Al}, \mathrm{Ti}, \mathrm{Ta}, \mathrm{Ni}_{3} \mathrm{Al}, \mathrm{Ni}_{3} \mathrm{Ta}$ and $\mathrm{Ni}_{3} \mathrm{Ti}$ [23].

\subsection{Geometry of the simulation supercell}

Supercells of $\mathrm{L}_{2}$ structure $(P m \overline{3} m$, atomic positions of $\mathrm{Ni}$ and $\mathrm{Al} / \mathrm{X}$ are $(0.5,0.5,0)$ and $(0,0,0)$ respectively) were constructed in two orientations for simulating $\mathrm{APB}_{(111)}$ and $\mathrm{APB}_{(010)}$.

For calculating the $\mathrm{APB}_{(111)}$ energy, the basis vectors of the supercell were oriented along the $\left[\begin{array}{lll}1 & -2 & 1\end{array}\right],\left[\begin{array}{lll}1 & 0 & -1\end{array}\right]$ and $\left[\begin{array}{lll}1 & 1 & 1\end{array}\right]$ crystallographic directions of $\mathrm{L}_{2}$. This supercell consisted of 6 identical $\{111\}$ layers stacked in a...-ABC-ABC-... manner, and wherein each layer is translated by a $1 / 6[1-21]$ vector with respect to the layer below. Each $\{111\}$ layer contains $6 \mathrm{Ni}$ sites and $2 \mathrm{Al}$ sites. Figure 1a shows the projection of atoms on the (111) plane. The circles, squares and triangles represent atoms on layer $\mathrm{A}, \mathrm{B}$ and $\mathrm{C}$ respectively.

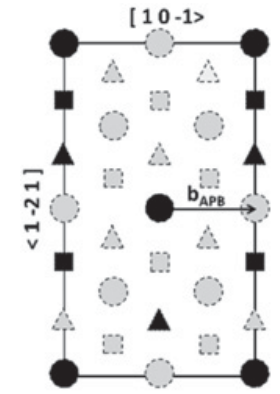

(a)

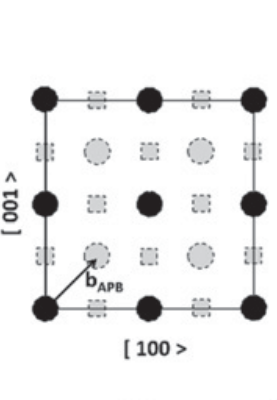

(b)

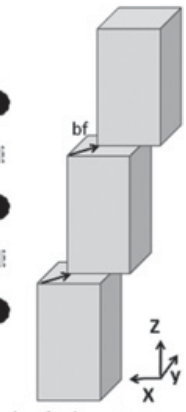

$b_{f}$, fault vector
Figure 1. Projection of atoms on a) close packed plane (111) and b) cube plane (010). The solid symbols in black represent atoms in Al-sublattice. The dashed symbols in grey represent Nisublattice. The schematic on the right shows "tiling" method used for creation of the fault. $\mathrm{b}_{\mathrm{APB}}=1 / 2[10-1]$.

The basis vectors of the supercell for $\mathrm{APB}_{(010)}$ were oriented along [100], [001] and [010] crystallographic directions. The supercell contains $6\{010\}$ layers that are stacked in $\mathrm{a} . .-\mathrm{ab}-\mathrm{ab}-\ldots$ manner. While layer "a" consists of $4 \mathrm{Ni}$ sites and $4 \mathrm{Al}$ sites, layer "b" has $8 \mathrm{Ni}$ sites. Figure $1 b$ shows the projection of atoms on (010) plane. The circles and squares represent atoms on layers "a" and "b" respectively. The number of atoms in both the $\mathrm{APB}_{(111)}$ and $\mathrm{APB}_{(010)}$ supercells were 48.

\subsection{Evaluation of APB energy}

APBs were created by the "tiling" technique wherein atomic positions are fixed with respect to the original supercell, but the supercell repeat vectors are chosen such that a planar fault is created on imposing periodic boundary conditions [32]. This method results in creation of one APB per supercell. The shear vector associated with the $\mathrm{APB}$ was $1 / 2\left[\begin{array}{lll}1 & 0 & -1\end{array}\right]$ for both $\mathrm{APB}_{(111)}$ and $\mathrm{APB}_{(010)}$ supercells. The energy of the stacking fault was calculated using the formula:

$$
\frac{E_{\text {faulted }}-E_{\text {perfect }}}{A}
$$

where $E_{\text {fault }}$ is energy of the faulted structure, $E_{\text {perfect }}$ is the energy of perfect structure and $A$ is the area of the faulted region in the supercell.

\subsection{Probing the effect of alloying}

For computing APB on either plane, substitutions of $\mathrm{X}$ $(\mathrm{X}=\mathrm{Ta}$ or $\mathrm{Ti}$ ) were made in the $\mathrm{Al}$ sublattice to obtain a range of compositions of $\mathrm{Ni}_{3} \mathrm{Al}_{(1-x)} \mathrm{X}_{x}, \quad(\mathrm{x}=0,0.25$, $0.5,1)$. The number of atoms of $X$ substituted depends on $\mathrm{x}$. The additional variable is the spatial distribution of $\mathrm{X}$ in the supercell. In this regard, two types of supercells were considered for a given composition and type of fault.

In the first type, $\mathrm{X}$ atoms were distributed such that, the composition across the fault plane was the same as the composition away from the fault. Within this constraint, several configurations were generated to mimic a random distribution of $X$. The average fault energy for any composition was calculated by a weighted average of the fault energy for each configuration in this composition. The weighting factor for any configuration is probability 

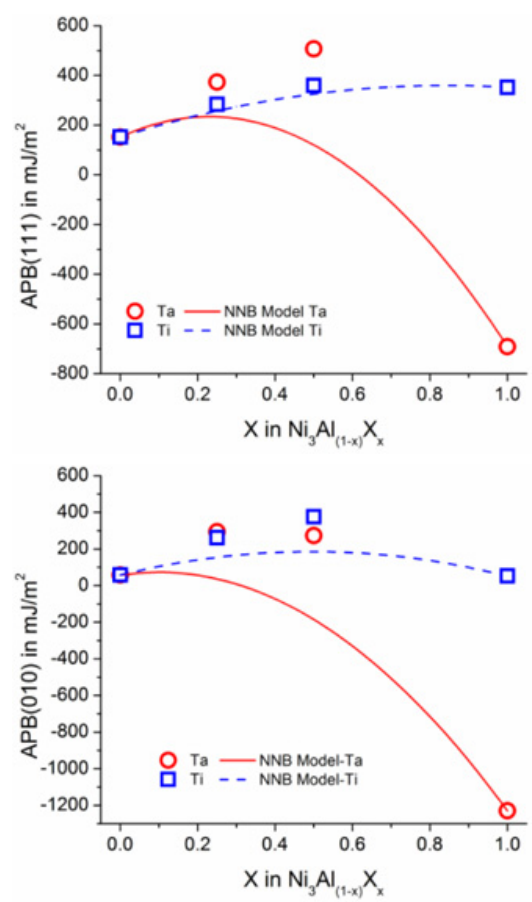

Figure 2. The variation of $\mathrm{APB}_{(111)}$ and $\mathrm{APB}_{(010)}$ as function of $x$, is shown for Ta and Ti substitutions. Also indicated via lines are the predictions of the NNB model.

of finding it in a random distribution of $\mathrm{X}$ atoms in $\mathrm{Al}$ sublattice. More details on this procedure will be published elsewhere [33]. These calculations will henceforth be referred to as homogeneous calculations.

In the second type of calculations, for a given alloy composition, $\mathrm{X}$ atoms were distributed in a nonuniform manner. As a consequence, the composition across the fault was different from the composition away from the fault. The composition across the fault was limited to three special cases: a) $\mathrm{Ni}_{3} \mathrm{Al}$, b) $\mathrm{Ni}_{3} \mathrm{Al}_{0.5} \mathrm{X}_{0.5}$, and c) $\mathrm{Ni}_{3} \mathrm{X}$. The composition away from the fault was adjusted such that the overall composition of the supercell corresponded to the desired composition of $\gamma^{\prime}$. These calculations will henceforth be referred to as heterogeneous calculations. Thus for each composition, three heterogeneous calculations are performed; one each with the composition on the fault being $\mathrm{Ni}_{3} \mathrm{Al}$, $\mathrm{Ni}_{3} \mathrm{Al}_{0.5} \mathrm{X}_{0.5}$, and $\mathrm{Ni}_{3} \mathrm{X}$. No averaging was done between these calculations and the fault energy for each of these heterogeneous calculations is individually reported. The reasons for doing this second type of calculation are explained in Section 3.3.

\section{Results and discussion}

\subsection{Effect of alloying on homogeneous fault energies}

The homogeneous $\mathrm{APB}_{(111)}$ and $\mathrm{APB}_{(010)}$ energies were derived by weighting the fault energy from various configurations for each composition of $\mathrm{Ni}_{3} \mathrm{Al}_{1-x} \mathrm{X}_{x}$. The variation of $\mathrm{APB}_{(111)}$ and $\mathrm{APB}_{(010)}$ as function of $x$, is shown for Ta and Ti substitutions in Fig. 2. It is observed that for both faults and for both alloying elements, fault energies initially increases with alloying and then decreases. While for $\mathrm{APB}_{(11)}$, the increase and decrease are steeper for Ta additions, for $\mathrm{APB}_{(010)}$ the increase is similar for $\mathrm{Ti}$ and $\mathrm{Ta}$, whereas the decrease is steeper for Ta. Indeed, the sharp decrease with Ta addition is manifested as negative $\mathrm{APB}_{(111)}$ and $\mathrm{APB}_{(010)}$ energies for $\mathrm{Ni}_{3} \mathrm{Ta}$. The observed trends are consistent with previous reports [20,23]. Vamsi et al. [23] have previously proposed a quasi-chemical model to predict APB energies in the dilute regime $(x<0.25)$. Here, we will review the model briefly and explore the applicability of the model for a wider range of compositions.

\subsection{Nearest neighbour bond model}

In a nearest neighbor bond model (i.e., a quasichemical approach), the energy of the system (either perfect or faulted) is considered as the the sum of bond energies between neighboring atoms. When an APB is created, while there is a no change in the stacking sequence, there is a change in environment at different distances. The creation of $\mathrm{APB}_{(111)}$ results in First Nearest Neighbor (FNN) and Second Nearest neighbor (SNN) violations for atoms on the atomic planes adjoining the fault [23]. These violations result in an energy penalty that contributes to the energy of the fault. There are also violations at the $4^{\text {th }}$ nearest neighbor distance and beyond, but it is reasonable to assume that their contribution to the fault energy would be negligible compared to the FNN and SNN violations. After the creation of the $\mathrm{APB}_{(111)}$, the net change in bonding is $\frac{1}{8}\left(B_{A l-A l}^{I}+B_{N i-N i}^{I}-2 B_{N i-A l}^{I}\right)$ per atom at the FNN distance and $-\frac{3}{8}\left(B_{A l-A l}^{I I}+B_{N i-N i}^{I I}-2 B_{N i-A l}^{I I}\right)$ per atom at the $\mathrm{SNN}$ distance [23]. In this notation, $B_{A l-A l}^{I I}$ (for instance) denotes an $\mathrm{Al}-\mathrm{Al}$ bond at the SNN distance. In the context of the nearest neighbor bond model:

$$
\begin{aligned}
\gamma_{A P B(111)}= & K^{I}\left(\phi_{A l-A l}^{I}+\phi_{N i-N i}^{I}-2 \phi_{N i-A l}^{I}\right) \\
& +K^{I I}\left(\phi_{A l-A l}^{I I}+\phi_{N i-N i}^{I I}-2 \phi_{N i-A l}^{I I}\right)
\end{aligned}
$$

where $\phi_{A l-A l}^{I}, \phi_{N i-N i}^{I}, \phi_{N i-A l}^{I}, \phi_{A l-A l}^{I I}, \phi_{N i-N i}^{I I}$ and $\phi_{N i-A l}^{I I}$ are the bond energies of $B_{A l-A l}^{I}, B_{N i-N i}^{I}, B_{N i-A l}^{I}, B_{A l-A l}^{I I}$, $B_{N i-N i}^{I I}$ and $B_{N i-A l}^{I I}$ and, $K^{I}$ and $K^{I I}$ are geometrical constants related to the number of violations at each distance and the number of atoms per unit area.

Using similar arguments, it can be shown that the creation of $\mathrm{APB}_{(010)}$, results in violations starting at the SNN distance. The net change in bonding on creation of $\mathrm{APB}_{(010)}$ is $-\frac{1}{8}\left(B_{A l-A l}^{I I}+B_{N i-A l}^{I I}-2 B_{N i-A l}^{I I}\right)$ per atom at the SNN distance [23] and:

$$
\gamma_{A P B(010)}=K^{I I *}\left(\phi_{A l-A l}^{I I}+\phi_{N i-N i}^{I I}-2 \phi_{N i-A l}^{I I}\right)
$$

where $K^{I I *}$ is a geometrical constant that is different $K^{I I}$ since this fault is on a different plane.

In a pseudo-binary system, where $\mathrm{X}$ substitutes $\mathrm{Al}$, in addition to Al-sublattice, Ni-Ni, Ni-Al, Ni-X, Al-Al, Al$\mathrm{X}, \mathrm{X}-\mathrm{X}$ bonds exist at different distances. The creation of the fault results in a change in bonding of all these types of bonds at the different distances. For instance, during the creation of $\mathrm{APB}_{(111)}$, two atoms initially in the Al sublattice in a perfect crystal are brought next to 


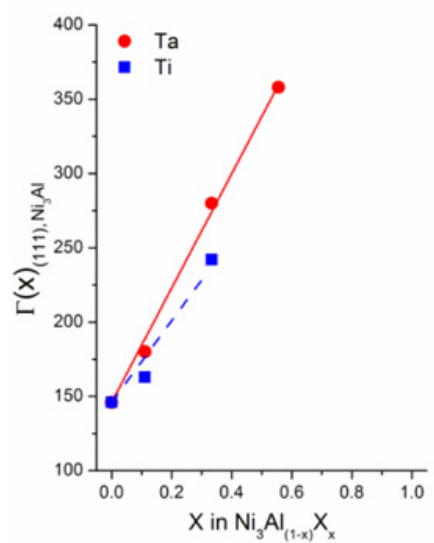

(a)

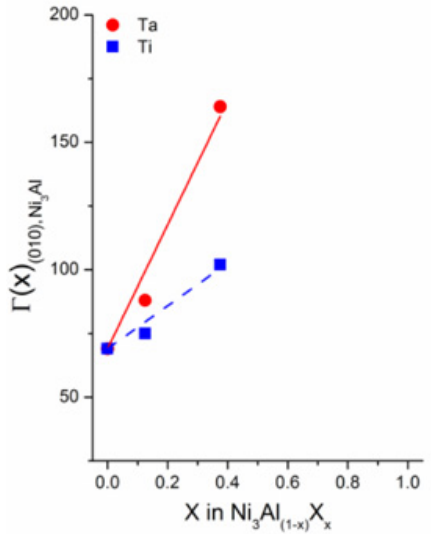

(d)

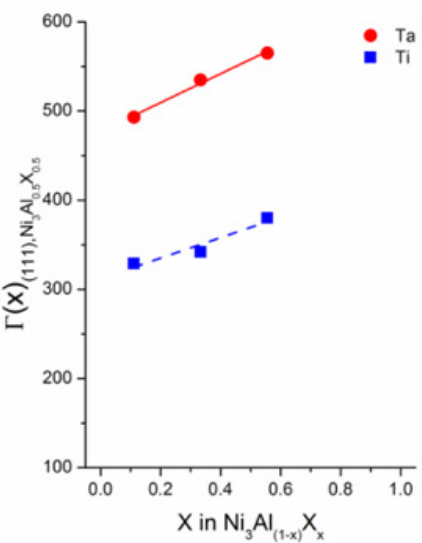

(b)

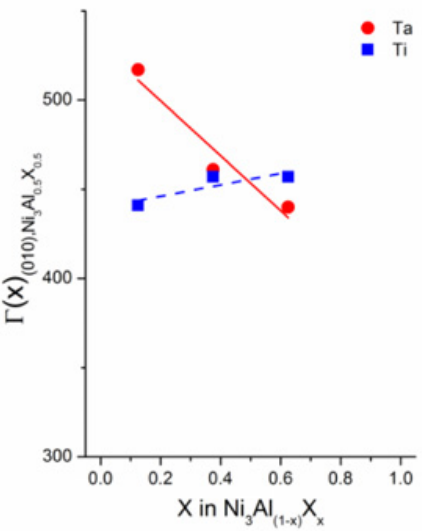

(e)

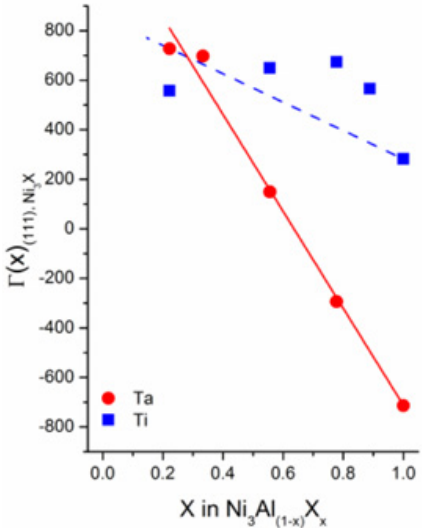

(c)

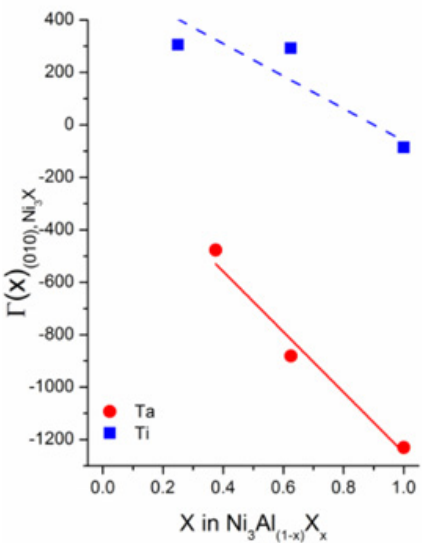

(f)

Figure 3. The effect of composition, (i.e. environment) on (a) $\Gamma(x)_{(111), N i_{3} A l}$, (b) $\Gamma(x)_{(111), N i_{3} A l_{0.5} X_{0.5}}$, (c) $\Gamma(x)_{(111), N i_{3} X},\left(\right.$ d) $\Gamma(x)_{(010), N i_{3} A l}$, (e) $\Gamma(x)_{(010), N i_{3} A l_{0.5} X_{0.5}}$ and (f) $\Gamma(x)_{(010), N i_{3} X}$.

each other at the first nearest neighbor distance. While in binary $\mathrm{Ni}_{3} \mathrm{Al}$, this results in the creation of $B_{A l-A l}^{I}$, in a pseudo-ternary system, it could result in the formation of $B_{A l-A l}^{I}, B_{A l-X}^{I}$ or $B_{X-X}^{I}$ bonds since the Al sublattice is also occupied by $X$. The relative numbers of these bonds is related to the amount of $\mathrm{X}$ in the $\mathrm{Al}$ sublattice (i.e., $x)$ and is given by $(1-x)^{2}: 2 x(1-x): x^{2}$. Additionally $\mathrm{APB}_{(111)}$ is also associated with the loss of $2 B_{N i-A l}^{I}$ bonds in a binary system. In a pseudo-binary system, losses are of $2(1-x) B_{N i-A l}^{I}$ bond and $2 x B_{N i-X}^{I}$ bonds. Similar arguments hold for SNN violations in $\mathrm{APB}_{(111)}$ as well. Thus, by appropriately weighting the probability of each type of violation, one can express the $\mathrm{APB}_{(111)}$ energy as:

$$
\begin{aligned}
& \gamma_{A P B(111)}=K^{I}\left[(1-x)^{2} \phi_{A l-A l}^{I}+2 x(1-x) \phi_{A l-X}^{I}\right. \\
& \left.\quad+x^{2} \phi_{X-X}^{I}+\phi_{N i-N i}^{I}-2(1-x) \phi_{N i-A l}^{I}-2 x \phi_{N i-X}^{I}\right] \\
& \quad+K^{I I}\left[(1-x)^{2} \phi_{A l-A l}^{I I}+2 x(1-x) \phi_{A l-X}^{I I}+x^{2} \phi_{X-X}^{I I}\right. \\
& \left.\quad+\phi_{N i-N i}^{I I}-2(1-x) \phi_{N i-A l}^{I I}-2 x \phi_{N i-X}^{I I}\right] .
\end{aligned}
$$

This can be reorganized into the following symmetrical form:

$$
\begin{aligned}
& \gamma_{A P B(111)}=K^{I}\left\{\left[(1-x)^{2}\left(\phi_{A l-A l}^{I}+\phi_{N i-N i}^{I}-2 \phi_{N i-A l}^{I}\right)\right]\right. \\
& +\left[2 x(1-x)\left(\phi_{A l-X}^{I}+\phi_{N i-N i}^{I}-\phi_{N i-A l}^{I}-\phi_{N i-X}^{I}\right)\right] \\
& \left.+\left[x^{2}\left(\phi_{X-X}^{I}+\phi_{N i-N i}^{I}-2 \phi_{N i-X}^{I}\right)\right]\right\}
\end{aligned}
$$

$$
\begin{aligned}
& +K^{I I}\left\{\left[(1-x)^{2}\left(\phi_{A l-A l}^{I I}+\phi_{N i-N i}^{I I}-2 \phi_{N i-A l}^{I I}\right)\right]\right. \\
& +\left[2 x(1-x)\left(\phi_{A l-X}^{I I}+\phi_{N i-N i}^{I I}-\phi_{N i-A l}^{I I}-\phi_{N i-X}^{I I}\right)\right] \\
& \left.+\left[x^{2}\left(\phi_{X-X}^{I I}+\phi_{N i-N i}^{I I}-2 \phi_{N i-X}^{I I}\right)\right]\right\} .
\end{aligned}
$$

Various prefactors can be collected and the equation may be rewritten in the following elegant manner:

$$
\begin{aligned}
& \gamma_{A P B(111)}=(1-x)^{2}\left(\Gamma_{(111), N i_{3} A l}\right)+2 x(1-x) \\
& \times\left(\Gamma_{(111), N i_{3} A l_{0.5} X_{0.5}}\right)+x^{2}\left(\Gamma_{(111), N i_{3} X}\right) \text {. }
\end{aligned}
$$

Where

$$
\begin{aligned}
& \Gamma_{(111), N i_{3} A l} \equiv K^{I}\left(\phi_{A l-A l}^{I}+\phi_{N i-N i}^{I}-2 \phi_{N i-A l}^{I}\right) \\
& +K^{I I}\left(\phi_{A l-A l}^{I I}+\phi_{N i-N i}^{I I}-2 \phi_{N i-A l}^{I I}\right) \\
& \Gamma_{(111), N i_{3} A l_{0.5} X_{0.5}} \equiv K^{I}\left(\phi_{A l-X}^{I}+\phi_{N i-N i}^{I}-\phi_{N i-A l}^{I}-\phi_{N i-X}^{I}\right) \\
& +K^{I I}\left(\phi_{A l-X}^{I I}+\phi_{N i-N i}^{I I}-\phi_{N i-A l}^{I I}-\phi_{N i-X}^{I I}\right) \\
& \Gamma_{(111), N i_{3} X} \equiv K^{I}\left(\phi_{X-X}^{I}+\phi_{N i-N i}^{I}-2 \phi_{N i-X}^{I}\right) \\
& +K^{I I}\left(\phi_{X-X}^{I I}+\phi_{N i-N i}^{I I}-2 \phi_{N i-X}^{I I}\right) \text {. }
\end{aligned}
$$

When the various terms in the equation are rewritten as in the manner shown in Eq. (6), it is evident that Eq. (6a) and Eq. (2) are identical. In other words, the term, $\Gamma_{(111), N i_{3} A l}$ in Eq. (6) is the $\mathrm{APB}_{(11)}$ energy in binary $\mathrm{Ni}_{3} \mathrm{Al}$. Similarly, 
given the symmetry between Eqs. (6a) and (6c), it is clear that $\Gamma_{(111), N i_{3} X}$ corresponds to $\mathrm{APB}_{(111)}$ energy in binary $\mathrm{Ni}_{3} \mathrm{X}$ The second term $\Gamma_{(111), N i_{3} A l_{0.5} X_{0.5} \text { corresponds }}$ to $\mathrm{APB}_{(111)}$ energy for a pseudo-binary $\mathrm{Ni}_{3} \mathrm{Al}_{0.5} \mathrm{X}_{0.5}$ in a configuration where APB creation results in the formation of only Al-X type interactions across the fault plane.

The NNB bond model can also be written for the $\mathrm{APB}_{(010)}$ energy in a similar manner. Note that the dependence of APB energy has a quadratic dependence on composition as used previously by Vamsi et al. [23]. Such a quadratic dependence of fault energy on composition has been also been used for modeling intrinsic stacking fault energy in a Fe-Cr-Ni solid solution [34]. The strength of the present model lies in the fact that it would potentially allow the determination of APB energy for any arbitrary composition of $\mathrm{X}$, in $\mathrm{Ni}_{3} \mathrm{Al}_{(1-x)} \mathrm{X}_{x}$ with the knowledge of only three terms, i.e., the APB energy for the terminal $\mathrm{Ni}_{3} \mathrm{Al}$ and $\mathrm{Ni}_{3} \mathrm{X}$ compounds and for one configuration of $\mathrm{Ni}_{3} \mathrm{Al}_{0.5} \mathrm{X}_{0.5}$. Using the three terms, which were already evaluated, the model was used to predict the energy of both APBs and for $\mathrm{X}=\mathrm{Ta}$ and Ti. The comparison between the prediction of the NNB model and the actual calculated value is shown in Fig. 2.

From Fig. 2, it is observed that while the NNB bond model is perhaps a reasonable model for predicting $\mathrm{APB}_{(111)}$ energy in $\mathrm{Ni}_{3}(\mathrm{Al}, \mathrm{Ti})$ system, it is unreliable for predicting $\mathrm{APB}_{(010)}$ energy in $\mathrm{Ni}_{3}(\mathrm{Al}, \mathrm{Ti})$ and energy of both APBs in $\mathrm{Ni}_{3}(\mathrm{Al}, \mathrm{Ta})$. As likely cause of this, is the fact that the nearest neighbor bond model is strictly applicable only in covalently bonded compounds where it may be possible to assign unique values of bond energies. The nearest neighbor bond model has been shown to be inaccurate for predicting other defect properties such as vacancy formation energies and surface energies, in metals. This is due to the fact that the strength of a pairwise interaction in a metal is strongly influenced by the local electron density, which in turn depends on the atomic environment in which the bond sits.

\subsection{Environment-dependent nearest neighbour bond (EDNNB) model}

We propose that NNB model is inadequate because the terms $\Gamma_{(111), \mathrm{Ni}_{3} \mathrm{Al}}, \Gamma_{(111), \mathrm{Ni}_{3} \mathrm{X}}$ and $\Gamma_{(111), \mathrm{Ni}_{3} \mathrm{Al}_{0.5} \mathrm{X}_{0.5}}$ are not constant, but instead dependent on composition (i.e., on the environment). The composition dependence of the terms leads to the following EDNNB model for $\mathrm{APB}_{(111)}$ :

$$
\begin{aligned}
\gamma_{A P B(111)}= & (1-x)^{2}\left(\Gamma(x)_{(111), N i_{3} A l}\right)+2 x(1-x) \\
& \times\left(\Gamma(x)_{(111), N i_{3} A l_{0.5} X_{0.5}}\right)+x^{2}\left(\Gamma(x)_{(111), N i_{3} X}\right) .
\end{aligned}
$$

A similar equation may be written for $\mathrm{APB}_{(010)}$ as well. Physically the term, $\Gamma(x)_{(111), N i_{3} A l}$ can be interpreted as the $\mathrm{APB}_{(111)}$ energy for a heterogeneous configuration where the composition on the fault plane is $\mathrm{Ni}_{3} \mathrm{Al}$, while the composition away from the fault contains both $\mathrm{X}$ and $\mathrm{Al}$ atoms, in a proportion such that the overall composition of the supercell is $\mathrm{Ni}_{3} \mathrm{Al}_{(1-x)} \mathrm{X}_{x}$. The other two terms can be similarly understood. It was in this context that the heterogeneous
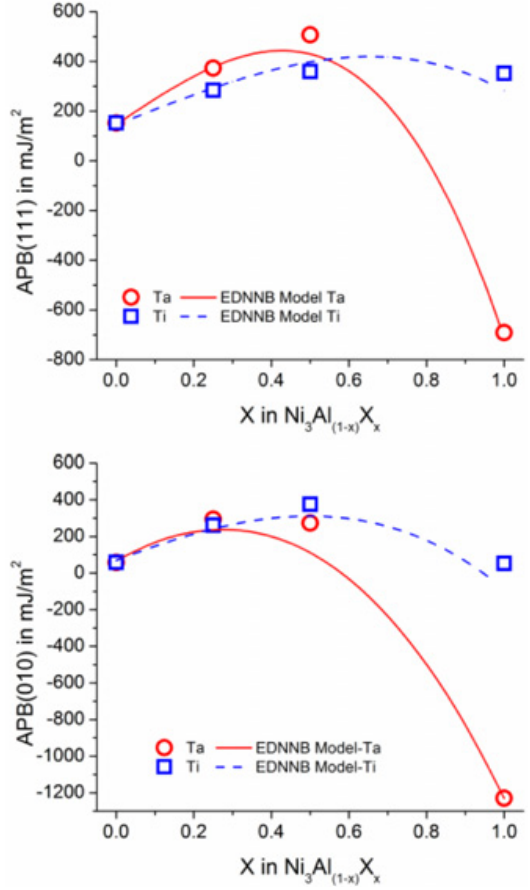

Figure 4. A comparison between the values predicted by EDNNB model and the calculated values of $\mathrm{APB}(111)$ and $\mathrm{APB}(010)$. The hollow red circles and blue squares represent Ta and Ti respectively. The improvement in the match over the NNB model (Fig. 2) is evident.

calculations, described in Section 2.4, were conducted. The result of these specific heterogeneous calculations are the three terms: $\Gamma(x)_{(111), N i_{3} A l}, \Gamma(x)_{(111), N i_{3} X}$ and $\Gamma(x)_{(111), N i_{3} A l_{0.5} X_{0.5}}$. It should be noted that for deriving $\Gamma(x)_{(111), N i_{3} A l_{0.5} X_{0.5}}$, atomic configuration should be such that, the composition on the fault is $\mathrm{Ni}_{3} \mathrm{Al}_{0.5} \mathrm{X}_{0.5}$ and only Al-X type FNN occur after creation of the fault. Figure 3 shows the effect of composition on $\Gamma(x)_{(111), N i_{3} A l}, \Gamma(x)_{(111), N i_{3} X}$ and $\Gamma(x)_{(111), N i_{3} A l_{0.5} X_{0.5}}$. These results confirm our hypothesis that $\Gamma_{(111), N i_{3} A l}$, $\Gamma_{(111), N i_{3} X}$ and $\Gamma_{(111), N i_{3} A l_{0.5} X_{0.5}}$ are not constant, but strongly dependent on composition for both faults and for $\mathrm{Ti}$ and $\mathrm{Ta}$ additions. For example, both ternary additions $\mathrm{Ta}$ and $\mathrm{Ti}$ increase $\Gamma(x)_{(111), N i_{3} \mathrm{Al}}$ and $\Gamma(x)_{(010), N i_{3} \mathrm{Al}}$, with the dependence being stronger in the case of Ta. These results imply that the penalty paid for $\mathrm{Al}-\mathrm{Al}$ and $\mathrm{Ni}-\mathrm{Ni}$ FNN violations (for example) depend on the composition. To the best of the author's knowledge, this is the first report of an environment effect on fault energies.

As a first order approximation, the dependence of $\Gamma$ on $x$ is assumed to be linear. Using the derived linear dependence of the various $\Gamma$ terms, one can predict the energy of both APBs and for $\mathrm{X}=\mathrm{Ta}$ and Ti. The comparison between the prediction of the EDNNB model and the actual calculated value is shown in Fig. 4. It is evident that the EDNNB predictions are significantly better than that of the NNB model, for both faults and both alloying additions. This also confirms the importance of accounting for the effect of far-field composition on the penalty paid for violations on the fault. It must be noted that in comparison to other methods of calculating fault energies [35] where the large supercells and several 
configurations are needed to accurately capture the effect of composition on fault energy, a significantly smaller number of simulations are required to evaluate the model parameters, i.e., $\Gamma(x)_{(111), N i_{3} A l}, \Gamma(x)_{(111), N i_{3} X}$ and $\Gamma(x)_{(111), N i_{3} A l_{0.5} X_{0.5}}$.

The EDNNB model can be improved by assuming a non-linear dependence of $\Gamma$ on $x$. The model is also easily extendable to other pseudo-binary and even pseudo-ternary systems, and research is currently underway to develop models for multi-component systems.

\section{Summary}

First principles calculations were done for $\mathrm{Ni}_{3} \mathrm{Al}_{1-x} \mathrm{X}_{x}$ systems $(X=T a, T i, x=0-1)$ to calculate $\mathrm{APB}_{(111)}$ and $\mathrm{APB}_{(010)}$. It was shown that bonding violations on the fault are affected by far-field composition. Incorporating these composition effects, an environment dependent nearest neighbor bond (EDNNB) model was developed which predicts fault energies that are in good agreement with calculated values.

The authors acknowledge the financial support of Defence Research and Development Organization (DRDO), Government of India. KVV acknowledges the $\mathrm{PhD}$ fellowship provided by University Grants commission (UGC), Government of India.

\section{References}

[1] C. T. Liu and D. P. Pope, Intermetallic Compounds. - Structural Appications of Intermetallic Compounds 55 (John Wiley \& Sons, Ltd, 2002)

[2] R. C. Reed, The Superalloys: Fundamentals and Applications (Cambridge University Press, 2008)

[3] B. Reppich, Acta Metall. 30, 87 (1982)

[4] B. Reppich, P. Schepp, G. Wehner, Acta Metall. 30, 95 (1982)

[5] G. B. Viswanathan, P. M. Sarosi, M. F. Henry, D. D. Whitis, W. W. Milligan, M. J. Mills, Acta Mater. 53, 3041 (2005)

[6] T. M. Pollock, A. S. Argon, Acta Metall. Mater. 40, 1 (1992)

[7] L. Kovarik, R. R. Unocic, J. Li, P. Sarosi, C. Shen, Y. Wang, and M. J. Mills, Prog. Mater. Sci. 54, 839 (2009)

[8] R. C. Reed, N. Matan, D. C. Cox, M. A. Rist, and C. M. F. Rae, Acta Mater. 47, 3367 (1999)

[9] B. H. Kear, H. G. F. Wilsdorf, Trans. Metall. Soc. AIME. 224, 382 (1962)
[10] E. Nembach, K. Suzuki, M. Ichihara, S. Takeuchi, Philos. Mag. A 51, 607 (1985)

[11] N. M. Rosengaard, H. L. Skriver, Phys. Rev. B 50, 4848 (1994)

[12] M. H. Yoo, C. L. Fu, J. A. Horton, Mater. Sci. Eng. A 176, 431 (1994)

[13] A. F. Voter, S. P. Chen, MRS Proceeding. 82, 175 (1986)

[14] Y. Mishin, Acta Mater. 52, 1451 (2004)

[15] J. Douin, P. Veyssière, P. Beauchamp, Philos. Mag. A 54, 375 (1986)

[16] T. Kruml, E. Conforto, B. Lo Piccolo, D. Caillard, J. L. Martin, Acta Mater. 50, 5091 (2002)

[17] H. P. Karnthaler, E. T. Mühlbacher, C. Rentenberger, Acta Mater. 44, 547 (1996)

[18] D. M. Dimiduk, J. Phys. III 1, 1025 (1991)

[19] A. Korner, Philos. Mag. A 58, 507 (1988)

[20] M. Chandran, S. K. Sondhi, Model. Simul. Mater. Sci. Eng. 19, 025008 (2011)

[21] N. Baluc, H. P. Karnthaler, M. J. Mills, Philos. Mag. A 64, 137 (1991)

[22] N. Baluc, R. Schäublin, K. J. Hemker, Philos. Mag. Lett. 64, 327 (1991)

[23] K. V. Vamsi, S. Karthikeyan, Proceeding of Twelfth International Symposium on Superalloys, 521 (The Minerals, Metals and Materials Society (TMS), Warrendale, PA, 2012)

[24] G. Kresse, J. Hafner, Phys. Rev. B 47, 558 (1993)

[25] G. Kresse, J. Furthmüller, Phys. Rev. B 54, 11169 (1996)

[26] G. Kresse, J. Hafner, Phys. Rev. B 49, 14251 (1994)

[27] G. Kresse, J. Furthmüller, Comput. Mater. Sci. 6, 15 (1996)

[28] MedeA version 2.6.6, Materials Design Inc, Angel Fire, NM, USA (2009)

[29] M. Methfessel, A. T. Paxton, Phys. Rev. B 40, 3616 (1989)

[30] J. P. Perdew, K. Burke, M. Ernzerhof, Phys. Rev. Lett. 77, 3865 (1996)

[31] J. P. Perdew, K. Burke, M. Ernzerhof, Phys. Rev. Lett. 78, 1396 (1997)

[32] V. Vitek, Cryst. Lattice Defects 5, 1 (1974)

[33] K. V. Vamsi, S. Karthikeyan (Unpublished)

[34] P. J. Ferreira, P. Müllner, Acta Mater. 46, 4479 (1998)

[35] P. J. H. Denteneer, W. van Haeringen, J. Phys. C Solid State Phys. 20, L883 (1987) 